\title{
Computational investigation of capillary absorption in concrete using a three-dimensional mesoscale approach
}

\author{
S. Dehghanpoor Abyaneh, H.S. Wong, and N.R. Buenfeld \\ Concrete Durability Group, Department of Civil and Environmental Engineering, Imperial College \\ London
}

* Corresponding author. Tel.: +44 (0)20 75941195.

E-mail address: sdehghan@imperial.ac.uk (S. Dehghanpoor Abyaneh).

\begin{abstract}
In many exposure environments, capillary absorption of water controls the rate of deterioration of concrete. This paper presents a three-dimensional numerical investigation of capillary absorption by treating concrete as a heterogeneous composite discretised into a cubic lattice. The lattice elements were considered as conductive "pipes" with transport properties assigned based on the phase they represent. The capillary absorption process was described by a non-linear diffusion equation, with the hydraulic diffusivity a non-linear function of the degree of saturation of the composite. A non-linear finite element method was used to solve the governing differential equations. The numerical results were validated against analytical approximations, as well as experimental data from the literature. A sensitivity analysis was then performed to evaluate the effect of heterogeneities produced by aggregate particles on the absorbed water profile and the sorptivity coefficient. It was found that water penetrates concrete in an uneven profile influenced by the amount, spatial distribution and shape of the aggregate particles. Sorptivity decreased when spherical aggregate particles were replaced with ellipsoidal particles due to the consequent increase in tortuosity of the cement paste. This effect increased with increase in aspect ratio and volume fraction of aggregate. However, the size of aggregate particle appears to have an insignificant influence.
\end{abstract}

Keywords: Concrete; Cement-based materials; Lattice-Network model; Unsaturated flow; Capillary absorption; Aggregate shape; Sorptivity

\section{Highlights:}

- Capillary absorption in concrete was simulated using a three-dimensional model.

- Simulations were verified by comparing with experimental results and analytical approximations.

- Effects of different variables influencing capillary absorption were investigated.

- Sorptivity decreased with an increase in the aspect ratio of the aggregate particles.

\section{Introduction}

Most deterioration processes that affect concrete structures (e.g. reinforcement corrosion, sulphate attack, frost damage and leaching) occur as a result of intrusion of water. Water may be the main agent causing distress or may act as the transporting medium for aggressive species such as chlorides and sulphates. Therefore, the durability of concrete structures depends largely on its ability to resist the transport of water and dissolved aggressive species. Permeation, diffusion and absorption are three main transport mechanisms in cementitious materials. Permeation is the flow of fluids through a material under a pressure gradient, while diffusion is the movement of substances due to a concentration gradient. Absorption can be considered as the process of water uptake in a partially saturated material by means of capillary suction.

Many concrete structures in service experience wetting and drying cycles and so are rarely fully saturated. For example, basements and foundations experience fluctuating groundwater, and parts of marine and coastal structures are subjected to the tidal zone or salt spray. In these environments, the ingress and accumulation of aggressive species are driven by the non-linear, capillary absorption process rather than a Fickian diffusion or pressure-induced flow. This nonlinearity comes from the 
dependence of moisture diffusivity on water content [1]. Obviously, a good understanding of moisture transport is important in order to assess performance, predict degradation and develop service life models and durability-based design codes. The performance of certain structures is dependent on the ability of concrete to remain watertight or provide a physical barrier to contaminants. Thus, it is highly desirable to be able to predict the depth of moisture penetration and solute concentration profile over time for a given concrete, condition and exposure environment.

Previous studies have been made to enhance our understanding concerning capillary absorption in cement-based materials. The approaches used can be categorised into experimental, analytical and numerical. Lab based transport testing has been performed for many years to gain a better understanding of different variables affecting capillary transport in cement-based materials [1-7]. Some researchers have proposed analytical approaches to model capillary absorption of water in porous materials. Hall et al. [8] analysed the absorption of water into porous material containing nonsorptive inclusions in terms of unsaturated flow theory and showed that sorptivity is reduced by a factor $1-1.25 \alpha+0.26 \alpha^{2}$, where $\alpha$ is the volume fraction of inclusions. Although this equation was developed for the dilute case, it was found to be in good agreement with experimental data for gypsum plaster containing 2 to $49 \%$ sand volume fraction. In another study, Lockington et al. [9] proposed an analytical solution to predict water penetration profiles for unidirectional absorption in a homogeneous media. In this approach, hydraulic diffusivity is modelled by a nonlinear function of the degree of saturation. The predicted water penetration profiles were shown to be accurate by comparison against two sets of published experimental data for mortars.

The tremendous increase in computational capabilities has strongly favoured the development of numerical simulations based on a more precise description of microstructure. Numerical simulations should be able to give a more accurate prediction of effective properties of multiphase materials with complex internal microstructures compared to analytical predictions [10-12]. However, despite the numerous numerical modelling studies focusing on diffusion in cement-based materials, capillary absorption has received little attention [13-20]. Sadouki \& van Mier [21] presented a flow model for simulating heat and mass transfer in heterogeneous materials. The material was discretised as a twodimensional regular triangular lattice where the lattice elements are considered as conductive pipes. The model was then used to study the influence of the interfacial transition zone and non-saturated porous aggregate on moisture flow in concrete. More recently, Wang \& Ueda [22] proposed a twodimensional lattice network model to predict water penetration into concrete. Concrete was idealised at the mesoscale as a three-phase composite consisting of coarse aggregate, mortar and ITZ. The distribution of absorbed water content was calculated from the sorptivity and porosity of the mortar and ITZ phases.

There are significant advantages (and challenges) in simulating the capillary absorption process in three-dimensions, but to the best of our knowledge, this has not been reported before. Therefore, the aim of this work is to develop a framework to calculate the distribution of absorbed water content at any elapsed time given initial boundary conditions. The model will focus at the mesoscale, whereby concrete is idealised as a porous media containing aggregate particles of a range of size, shape and volume fraction, discretised into a lattice network. Water uptake by capillary absorption is described by a non-linear diffusion equation based on unsaturated flow theory, which is solved using a finite element method. A parametric study is then performed to evaluate the effect of heterogeneities produced by aggregate particles on the absorbed water profile and the sorptivity coefficient.

\section{Unsaturated flow}

This section provides a brief summary of the governing equations in the theory of unsaturated flow. For a more comprehensive treatment of the topic, readers are referred to previous reviews, for example by Hall $[1,23]$. Single-phase flow via capillary absorption in an unsaturated porous media is described by the extended Darcy equation:

$$
\boldsymbol{q}=-K(\theta) \nabla \boldsymbol{\Psi}(\theta)
$$

where $\boldsymbol{q}$ is the vector flow velocity, $K$ is the hydraulic conductivity [L/T] and $\boldsymbol{\Psi}$ is the capillary potential $[\mathrm{L}] . \theta$ is the reduced water content defined as: 


$$
\theta=\frac{\Theta-\Theta_{i}}{\Theta_{s}-\Theta_{i}}
$$

where $\Theta$ is the volumetric water content (i.e. ratio of volume of water to bulk volume of sample). $\Theta_{i}$ and $\Theta_{s}$ are the initial and saturated volumetric water contents, respectively. Thus, $\theta$ equals zero initially and one at saturation.

It is often more convenient to express capillary absorption (Eq. 1) in the form of a non-linear diffusion equation. This is obtained by combining Eq. (1) with the continuity equation and then rewriting the resultant equation in terms of $\theta$ by using the substitution $D(\theta)=K(\theta)(d \boldsymbol{\Psi} / d \theta)$, giving the equation:

$$
\frac{\partial \theta}{\partial t}=\nabla \cdot[D(\theta) \nabla \theta]
$$

The hydraulic diffusivity $D\left[\mathrm{~L}^{2} / T\right]$ is a moisture and temperature dependent parameter, and is considered as the fundamental material property that describes the process of capillary absorption. Applying the Boltzmann transformation to Eq. 3 and integrating the resulting equation gives the following expression for $D(\theta)$ :

$$
D(\theta)=-\frac{1}{2} \frac{1}{(d \theta / d \phi)_{\theta}} \int_{0}^{\theta} \phi d \theta
$$

Here, $\phi$ is the Boltzmann variable $\left(=x / t^{1 / 2}\right)$ where $x$ is the depth of water penetration and $t$ is the elapsed time. $D(\theta)$ can be estimated by measuring water content versus distance profiles after various elapsed times using non-destructive test methods such as NMR or $\mathrm{y}$-ray attenuation. However, obtaining accurate water penetration profiles in cement-based materials is difficult and this is not commonly carried out. A simpler approach to determine $D(\theta)$ is through an approximation using an exponential function of $\theta$ :

$$
D(\theta)=D_{0} e^{n \theta}
$$

in which $D_{0}$ is the limiting magnitude term and $n$ is the shape term [24]. This exponential law has been shown to be valid for a range of construction materials including soils, brick, stone, gypsum and concrete. It has also been found that $n$ varies little between materials, typically ranging from 6 to 8 [1, 24-27]. Lockington et al. [9] showed that $D_{0}$ can be obtained from the conventional sorptivity test using the following equation

$$
D_{0}=\frac{S^{2}}{\left(\Theta_{s}-\Theta_{i}\right)^{2}\left(e^{n}\left(2 n^{-1}-n^{-2}\right)-n^{-1}+n^{-2}\right)}
$$

In the sorptivity test, a cylindrical sample is initially dried to a uniform moisture state and then a flat surface is exposed to free water (Fig.1). The curved side surface is typically sealed with a waterproof coating (tape or epoxy) so that the uptake of water is approximately unidirectional in this arrangement. Gravitational effects are insignificant since water absorption in cement-based materials is dominated by capillary forces, at least for the short penetration distances during which sorptivity is measured. When these conditions are met, the cumulative mass of water absorbed per cross-section area $I$ scales to $t^{1 / 2}$ and the sorptivity $S$ can be determined from the slope of the best-fit line of:

$$
I=S t^{1 / 2}+A
$$

where $A$ is a small constant arising from minor surface effects in the experiment. 


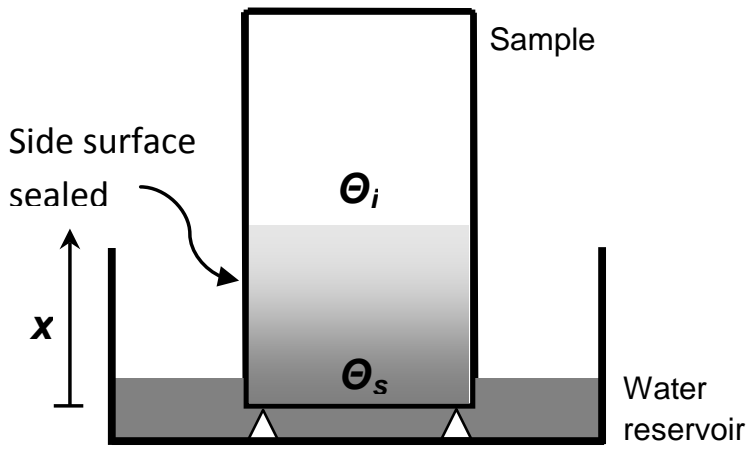

Fig. 1 Schematic of the sorptivity test.

\section{Methodology and description of the model}

In order to model transport phenomena in any material, one needs a porous structure as an input coupled with a transport algorithm. Our adopted method operates in two main steps. First, a threedimensional mesostructure of concrete is generated by randomly placing aggregate particles in the computational cube with the largest particles first with no overlaps. The aggregate particles follow a size distribution that is determined either from sieve analysis or generated from a theoretical gradation such as Fuller-Thompson [28]. A periodic boundary condition is applied to all sides of the computational cube. In doing so, aggregate particles that extend beyond the cube are completed (wrapped) into the opposite face of the cube.

In order to examine the effect of aggregate shape on sorptivity, ellipsoidal aggregate particles are incorporated into the model. An ellipsoid is defined by the following equation:

$$
(x-v)^{T} A^{-1}(x-v)=1
$$

Where $v$ is a vector representing the centre of the ellipsoid and $A$ is a positive definite matrix with eigenvectors which are the principal directions of the ellipsoid and eigenvalues which are the squares of the semi-axes: $a^{2}, b^{2}$ and $c^{2}$.

The mesostructure is projected into a regular lattice, so collision and overlap detection is a straightforward exercise of finding out whether two particles occupy the same site(s), rather than having to compute and test intersections between any two particles, which is usually very computationally expensive for non-spherical particles [29]. By varying the ratio $a: b: c$, four distinctive shapes ranging from spheres to elongated and disc shaped particles can be obtained. Table 1 shows the types of ellipsoids that will be used to represent aggregate particles in this study. Note that the ratios $a: b: c$ were selected such that the particle volume $(=4 / 3 \pi a b c)$ remains constant.

\section{Table 1 Shapes used to represent aggregate particles}

\begin{tabular}{lll}
\hline Shape & \multicolumn{1}{c}{$(a: b: c)$} \\
\hline Spherical & $(a=b=c)$ & $(1: 1: 1)$ \\
Tri-axial ellipsoidal & $(a>b>c)$ & $(1.5: 1: 0.67),(2: 1: 0.5)$ \\
Prolate spheroidal & $(a=b<c)$ & $(0.82: 0.82: 1.5),(0.71: 0.71: 2)$ \\
Oblate spheroidal & $(a=b>c)$ & $(1.5: 1.5: 0.44),(2: 2: 0.25)$ \\
\hline
\end{tabular}

Fig. 2 shows examples of randomly generated mesostructure containing spherical and non-spherical aggregate particles. Here, concrete is treated as a composite consisting of coarse aggregate particles and mortar. A computational cube of $50 \times 50 \times 50 \mathrm{~mm}^{3}$ at $0.5 \mathrm{~mm}$ resolution (voxel size) is used in all simulations. The aggregates simulated in this study will have sizes ranging from 5 to $20 \mathrm{~mm}$. Thus, 
the computational cube is at least 2.5 to 10 times the size of the aggregate particle and the aggregate particle is at least 10 to 40 times the size of the voxel. Each simulated mesostructure contains up to four hundred aggregate particles. It should be noted that because the mesostructure is randomly generated, each realisation of the same concrete differs in the placement of the aggregate particles. To obtain the sorptivity of a particular concrete, the simulation of water absorption is repeated for six random mesostructures and the results averaged to reduce the influence of size effects and statistical fluctuations. Our previous work on diffusivity [15] suggests that the sample size, resolution and averaging (number of random mesostructures) used is sufficient to obtain representative results.

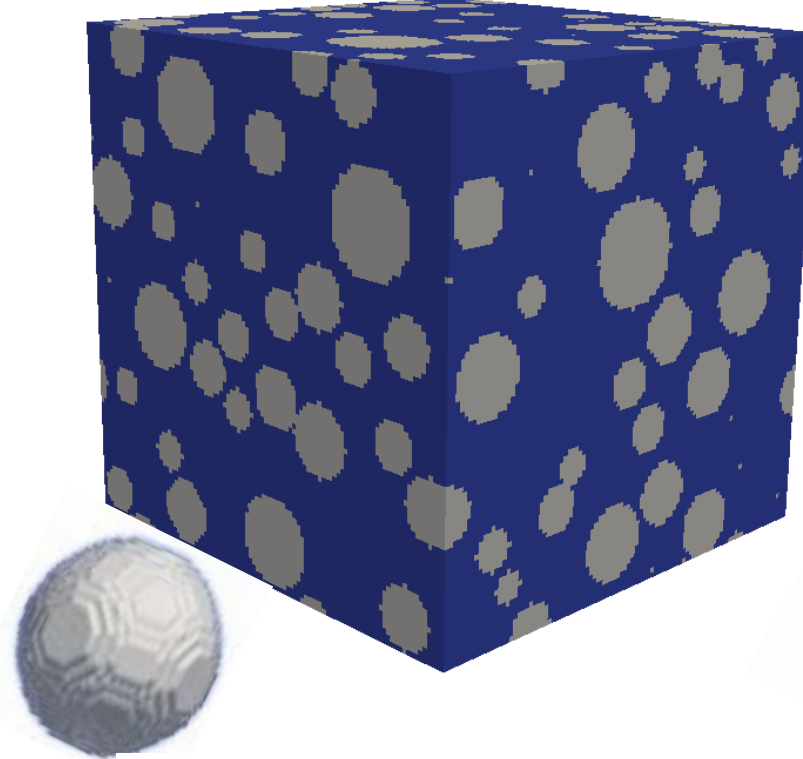

a) Spherical aggregate $(a: b: c=1: 1: 1)$

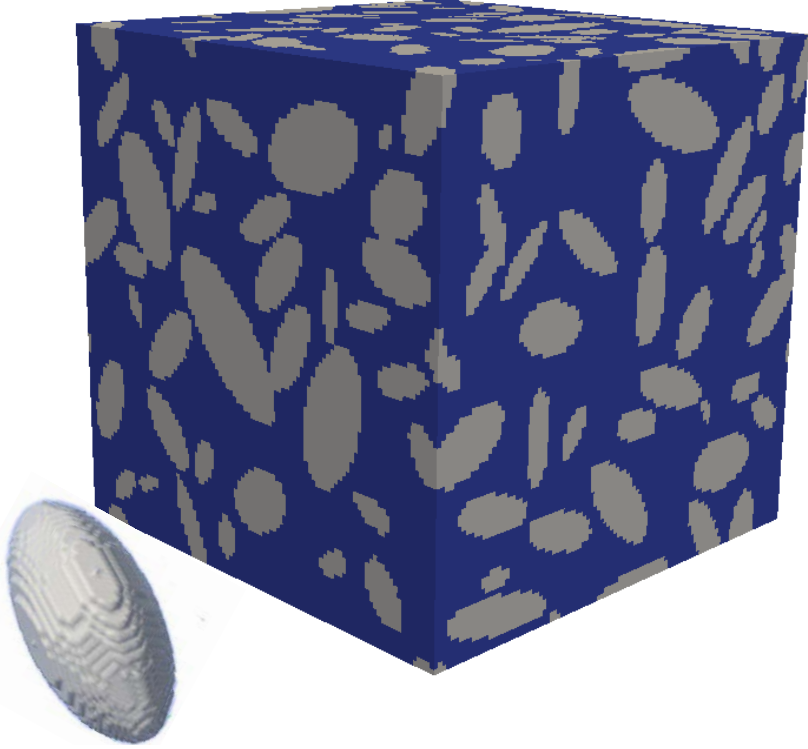

b) Tri-axial ellipsoidal aggregate $(a: b: c=2: 1: 0.5)$

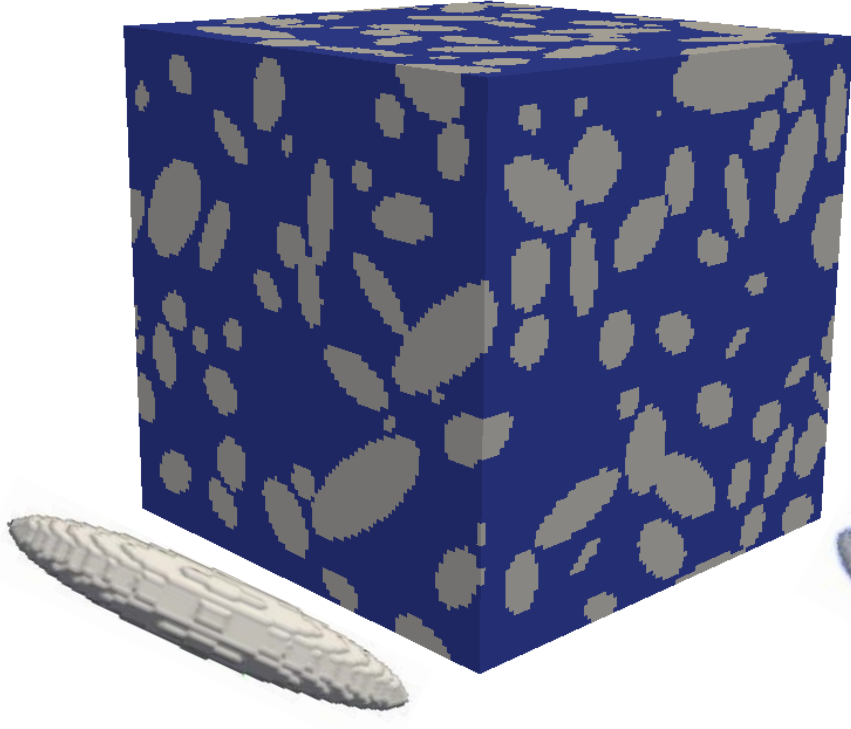

c) Prolate spheroidal aggregate $(a: b: c=0.71: 0.71: 2)$

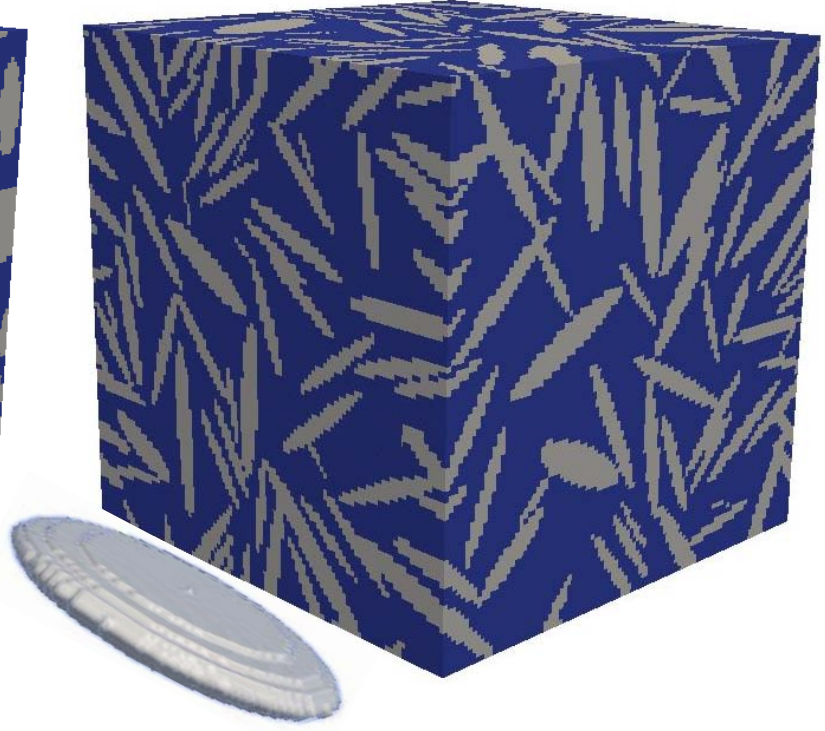

d) Oblate spheroidal aggregate $(a: b: c=2: 2: 0.25)$

Fig. 2 Randomly generated mesostructure containing $30 \%$ volume of spherical and nonspherical aggregate particles ranging from 5 to $10 \mathrm{~mm}$ (Fuller-Thompson gradation) in a $50 \times 50 \times 50 \mathrm{~mm}$ computational cube (voxel size $=0.5 \mathrm{~mm}$ ).

The second step is to apply an appropriate transport algorithm to the mesostructure. In the present paper, a lattice-type network model is established to simulate water absorption in concrete. The lattice-type network model is similar to that of Sadouki \& van Mier [21], but ours will be implemented on a three-dimensional mesostructure. The lattice elements are considered as conductive "pipes" 
connecting the centres of adjacent voxels, thus producing a cubic lattice network. The transport properties of lattice elements are assigned based on the phase they represent. As previously mentioned, the aggregates are assumed to be non-sorptive and consequently do not contribute to transport. Fig. 3 shows a two-dimensional schematic diagram illustrating how the lattice network is generated from the digitized mesostructure. Black voxels represent aggregate particles and white voxels represent the porous matrix. The pipe elements connect the centres of the white voxels. The material property of the porous matrix is assigned to these pipe elements. As the aggregate particles are assumed to be non-sorptive, there are no pipe elements connecting the matrix voxels to aggregate voxels or within the aggregate voxels.

A semi-discrete form of the capillary absorption process (Eq. 3) can be obtained using the Galerkin method as follows:

$$
\frac{A D(\theta)}{L}\left[\begin{array}{cc}
1 & -1 \\
-1 & 1
\end{array}\right]\left(\begin{array}{c}
\theta_{i} \\
\theta_{j}
\end{array}\right)+\frac{A L}{6 \mu}\left[\begin{array}{ll}
2 & 1 \\
1 & 2
\end{array}\right]\left(\begin{array}{c}
\frac{\partial \theta_{i}}{\partial t} \\
\frac{\partial \theta_{j}}{\partial t}
\end{array}\right)+A D(\theta)\left(\begin{array}{l}
\frac{\partial \theta}{\partial x} \mid x=x_{i} \\
\frac{\partial \theta}{\partial x} \mid x=x_{j}
\end{array}\right)=\left(\begin{array}{l}
0 \\
0
\end{array}\right)
$$

in which $A$ is the cross-section area of the lattice element, $D(\theta)$ is the moisture dependent hydraulic diffusivity, $L$ is the length of the lattice element, $\theta_{i}$ and $\theta_{i}$ are the moisture potential at nodes $i$ and $j$, respectively. $\mu$ is set to $1.0,2.0$ or 3.0 for $1-d, 2-d$, and $3-d$ networks, respectively [30].

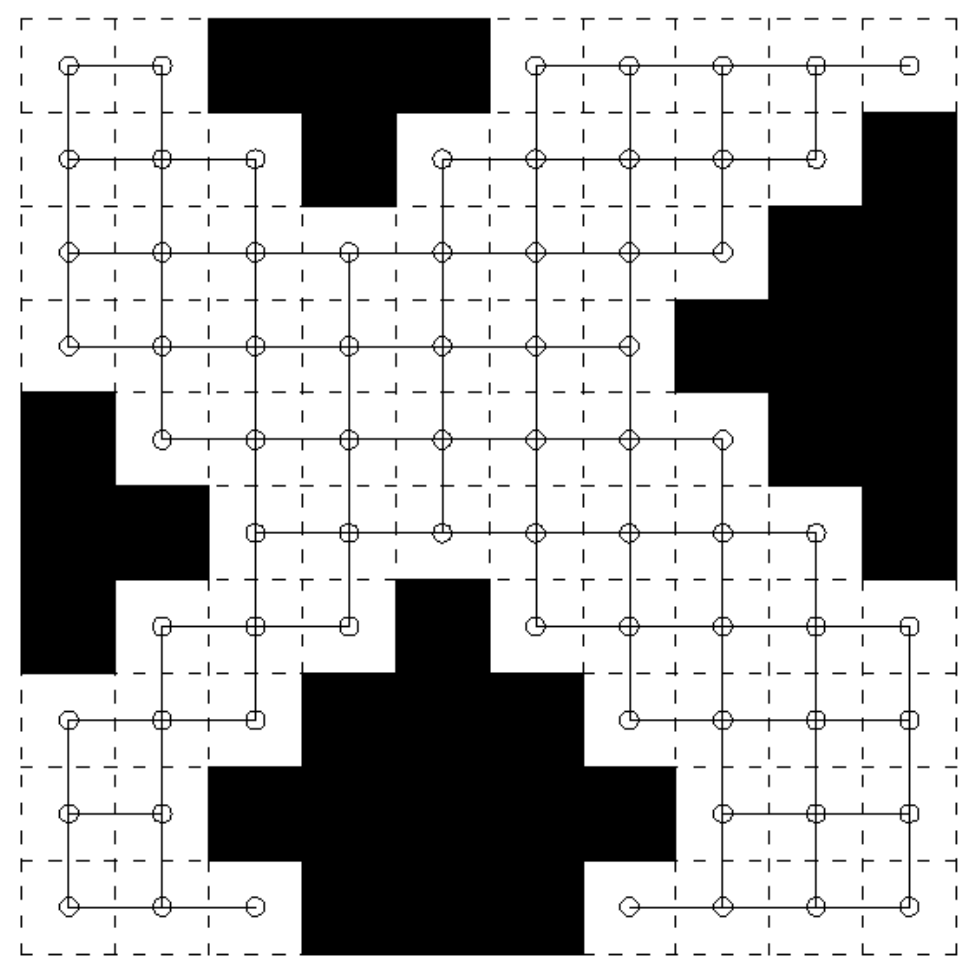

Fig. 3 Two dimensional schematic diagram of the digitized mesostructure to lattice network mapping. Dashed lines show the border of voxels. Black voxels represent aggregate particles. Solid lines connecting the centres of white voxels are the pipe elements of the lattice model.

To mimic capillary absorption into the mesostructure, the relative water content of all nodes in contact with free water is assigned to one. Thus, the Dirichlet boundary condition is imposed to solve the set of differential equations. Then, the Crank-Nicolson scheme is used for the solution of Eq. 9. Given the initial water content of all the nodes in the mesostructure, a system of equations is set up at each time step, and then solved to determine the new nodal water contents. It should be noted that within the Crank-Nicolson scheme, iterations are performed to capture the nonlinear dependence of hydraulic 
diffusivity $D$ on $\theta$. This enables us to calculate the water content distribution in the mesostructure as a function of space and time, given knowledge of the hydraulic diffusivity.

\section{Validation}

In order to validate the model, we compare our numerical solution with experimental data obtained by Hall [1] on a mortar bar made of 1:3:12 OPC/lime/sand by volume. In the experiment, water content distribution in the mortar bar $(35 \times 35 \times 235 \mathrm{~mm})$ was measured using NMR imaging at a series of elapsed times after one end of the bar was exposed to a water reservoir. The mortar was initially dried $\left(\Theta_{i}=0\right)$. The sorptivity and saturated water content of the mortar were $2.57 \mathrm{~mm} / \mathrm{min}^{0.5}$ and 0.27 respectively.

In addition, we compare our numerically predicted water penetration profiles with an approximate analytical solution by Lockington et al. [9] for the case of unidirectional water absorption in a homogeneous media. In the analytical solution, hydraulic diffusivity $D(\theta)$ is modelled by the exponential relationship shown in Eq. 5 where $D_{o}$ is calculated from sorptivity (Eq. 6) and the exponential $n$ is taken to vary between 6 to 8 . Once $D(\theta)$ is known, water penetration profiles are predicted using the following equation:

$$
2 D_{0}\left[E_{i}(n)-E_{i}(n \theta)\right]=s \phi+\frac{A}{2} \phi^{2}
$$

where $E_{i}(x)$ is the exponential integral $\left(E_{i}(x)=-\int_{-x}^{\infty} e^{-t} /{ }_{t} d t\right), s$ is the scaled sorptivity $(s=$ $\left.S /\left(\Theta_{S}-\Theta_{i}\right)\right)$ and $A$ is given by:

$$
A=\frac{e^{n} n^{-1}-1-n^{-1}}{e^{n}-1}
$$

We note that Eq. (10) is an approximate solution to the nonlinear diffusion equation that governs capillary absorption (Eq. 3) and that it has been shown to give reasonably accurate agreement to measured water penetration profiles for mortars when $n$ is taken as 6 [9].

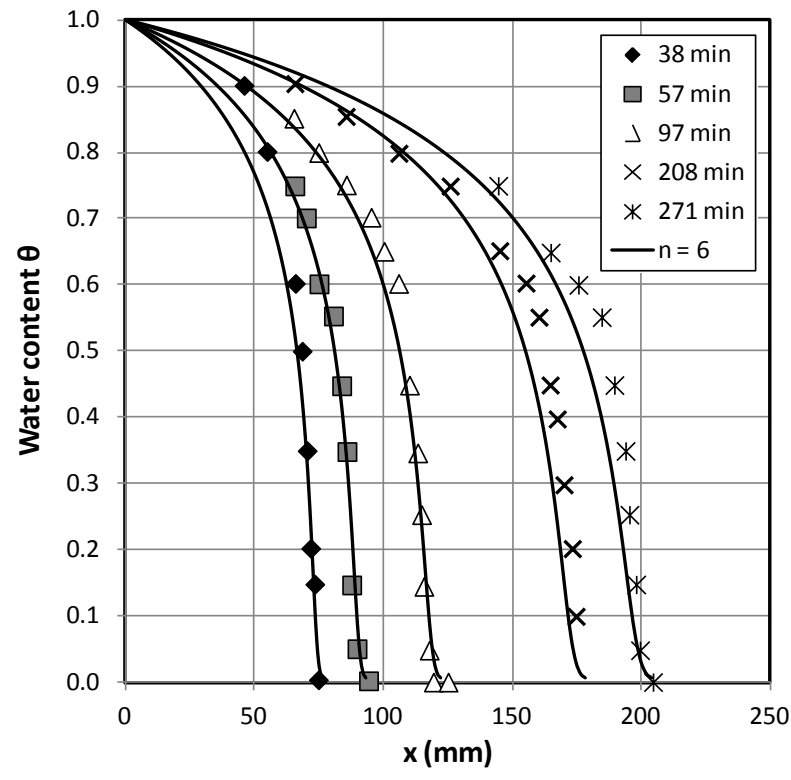

a) $n=6$

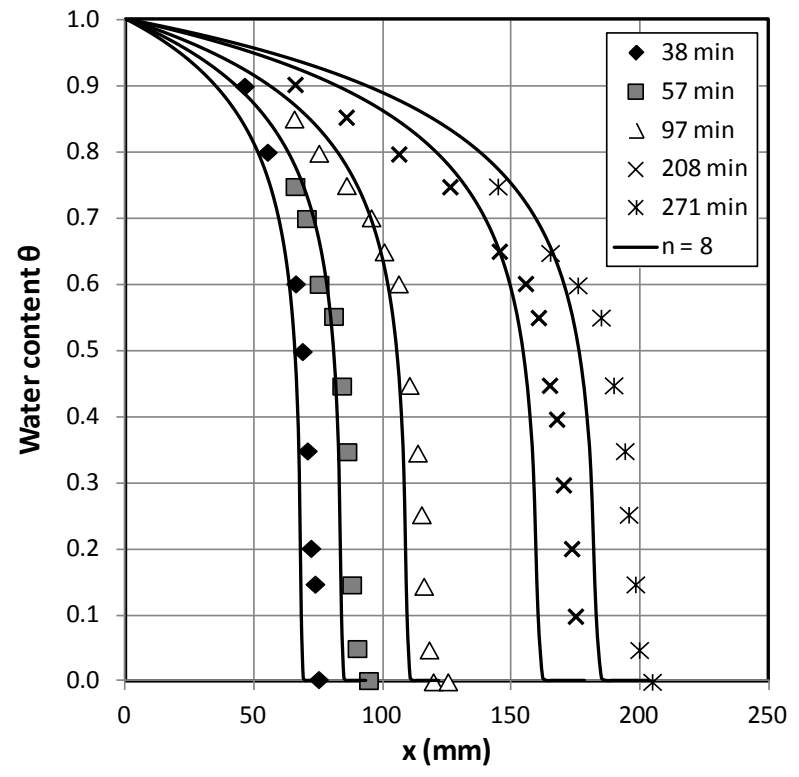

b) $n=8$ 


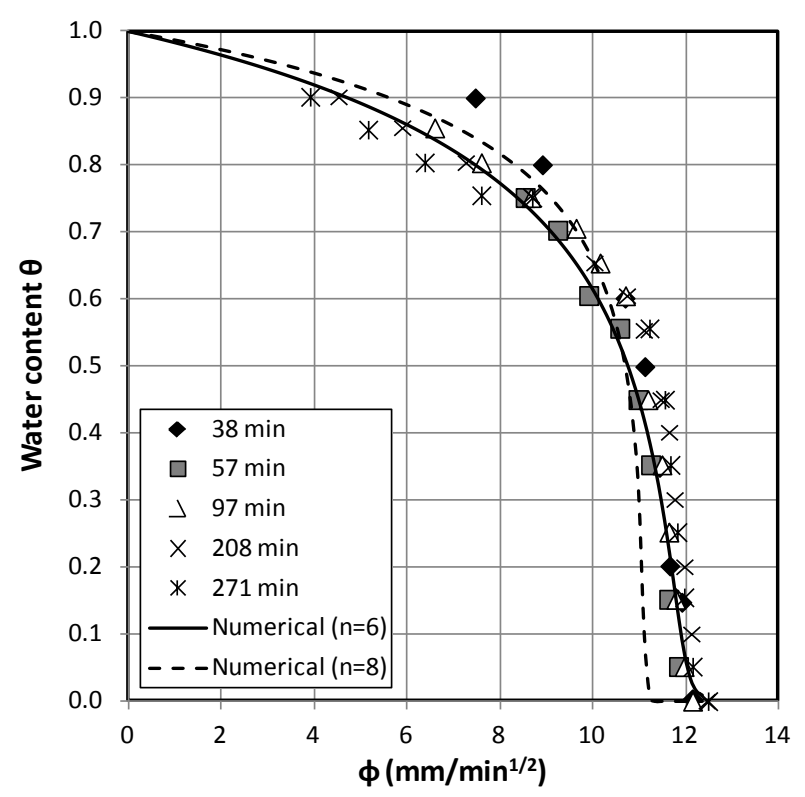

c) Water content versus Bolzmann variable $\left(\phi=x / t^{1 / 2}\right)$

Fig. 4 Comparison of water penetration profiles measured by Hall [1] against our numerical solution for a mortar with sorptivity of $2.57 \mathrm{~mm} / \mathrm{min}^{1 / 2}$ and saturated porosity of 0.27 .

The measured and simulated water penetration profiles are compared in Fig. 4 and Fig. 5. For ease of comparison with the analytical solution of Lockington et al. [9], we have simulated the penetration profile for a mortar that has the same sorptivity and porosity as the one tested by Hall [1]. It can be seen that the simulated profile shows a characteristic steep gradient near the wetting front consistent with experimental data. As expected, the water front advances along the sample with square-root of elapsed time while maintaining a steep fronted profile. Plotting in the form of the Boltzmann variable converges the profiles into a single 'master' curve (Fig. 4c) consistent with measurements and theory. The area under the curve is the scaled sorptivity of the sample.

The results show very good agreement between the analytical and numerical simulations, thus validating our numerical code. Our simulations also produced reasonable agreement with experimental data. It can be seen that changing the exponential $n$ between 6 and 8 produces only a small difference in the profile, however $n=6$ appears to give a better fit, particularly at longer absorption times. For $n=6$, the $D_{o}$ for this particular mortar is calculated to be $0.736 \mathrm{~mm}^{2} / \mathrm{min}$ This compares well with the best-fit results from Hall's data [1], where the parameters $n$ and $D_{0}$ were found to be 6.55 and $0.49 \mathrm{~mm}^{2} / \mathrm{min}$ respectively.

We should note that the analytical solution of Lockington et al. [9] was derived for a homogeneous media. The advantage of our numerical model compared to this approach is that more than one phase is possible, allowing the simulation of non-homogeneous media. In the next section, we present the results of our numerical simulations when applied to a three-dimensional concrete mesostructure to examine the effect of heterogeneities (here produced by aggregate particles) on the simulated water absorption profiles. 

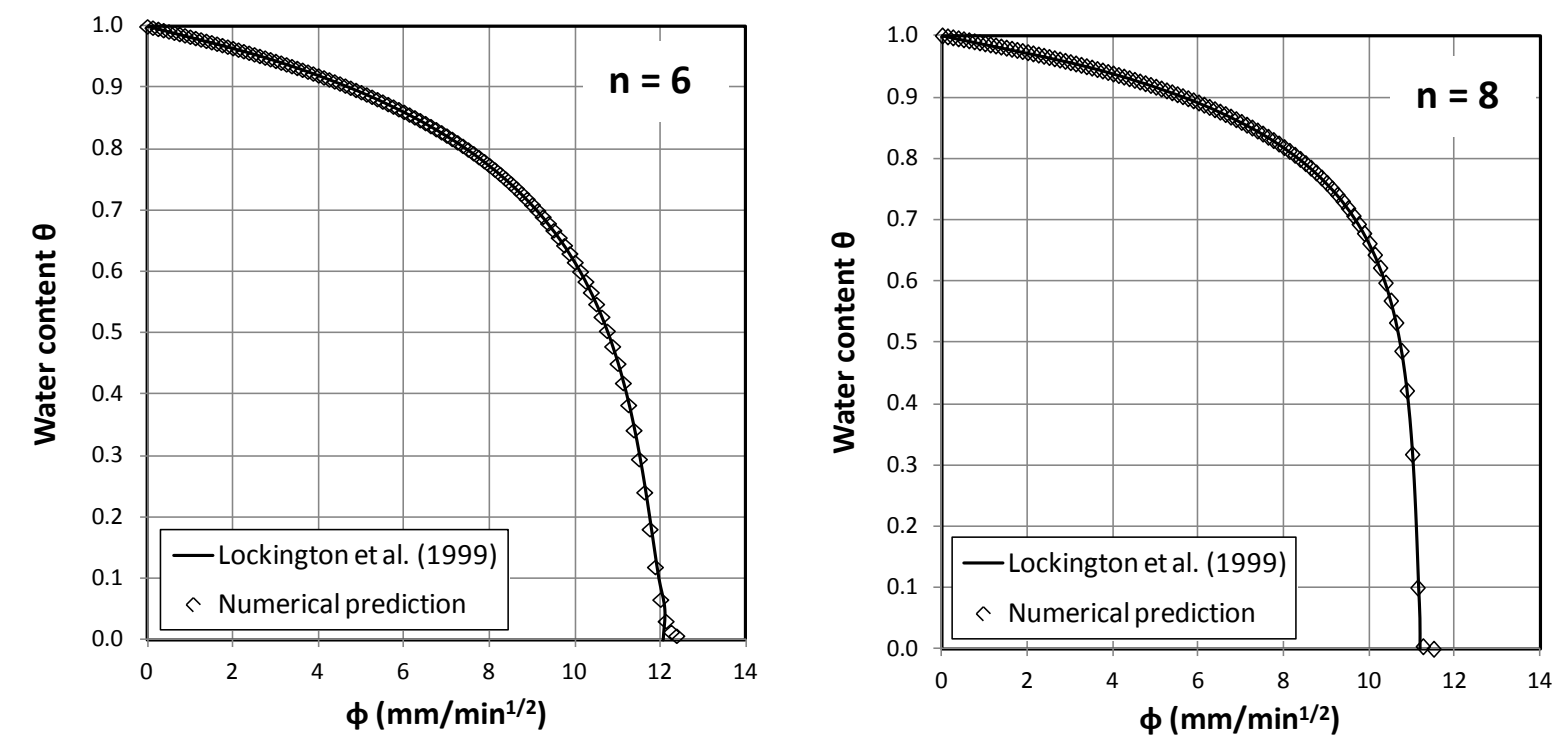

Fig. 5 Comparison of water penetration profile estimated from the analytical solution of Lockington et al. [9] against our numerical solution for a mortar with sorptivity of 2.57 $\mathrm{mm} / \mathrm{min}^{1 / 2}$ and saturated porosity of 0.27 (left: $\mathrm{n}=6$, right: $\mathrm{n}=8$ ).

\section{Results}

\subsection{Size and statistical effects}

Our numerical model is inevitably subject to effects of digital resolution, finite sample size and statistical fluctuation. Size effects arise since the physical volume of the numerical sample has been limited to speed up computation, while statistical effects are due to the random nature of the model. For example, at the start of each simulation the aggregate particles are randomly placed in the media, therefore the created mesostructure is different for each realisation. In order to decrease the effect of statistical fluctuations and the size effect, simulations are repeated and averaged. The reported sorptivity results are the average of six different realisations. Overall, we found that the sorptivity values have a coefficient of variation (standard deviation/average) of less than $4 \%$. Furthermore, the isotropy of the samples was examined by computing the sorptivity in each of the three directions. The standard deviation is again less than $4 \%$.

The resolution in the digitized mesostructure may also affect the accuracy of the simulations. In general, the accuracy of the simulations should increase with higher resolution. However, the available computational resource imposes a practical limit on the resolution to be used. It was found that the sorptivity values differ by less than $2 \%$ when the resolution (voxel size) was increased from $0.5 \mathrm{~mm}$ to $0.25 \mathrm{~mm}$. On a single 64-bit PC (3.4 GHz, 8GB RAM), the simulations take a few hours to converge depending on the aggregate volume fraction. The simulation time increased by more than 8 times when resolution (voxel size) was increased from $0.5 \mathrm{~mm}$ to $0.25 \mathrm{~mm}$. Thus, it was concluded that a $0.5 \mathrm{~mm}$ resolution gives reasonably accurate results at an acceptable computational cost.

We then evaluated the effect of $D_{0}$ and $n$ on the simulated sorptivity ratio $S / S_{m}$, where $S$ and $S_{m}$ are the sorptivity of the concrete and mortar respectively. A set of simulations was performed where the scaled sorptivity for mortar $s$ was varied between 1 and $10 \mathrm{~mm} / \mathrm{min}^{1 / 2}$. This covers a wide range of experimental sorptivity data for mortars of varying formulations and maturity $[1,2]$. Then, $D_{0}$ was calculated as a function of $n(6-8)$ using Eq.6. It was found that the effect of $D_{0}$ and $n$, and thus the choice of $s$ on the simulated sorptivity ratio $S / S_{m}$ for concrete, is negligible.

In the following sections, simulations are performed using the proposed model to examine the relative influence of aggregate volume fraction, size and particle shape on the water penetration profile and sorptivity of concrete. In all cases, the numerical sample is a cube of $50 \mathrm{~mm}$ divided to 100 regular voxel elements in each direction (Fig. 2). We consider the case of water absorption into concrete which is initially dried and then subject to a free water boundary condition. For the ease of comparison with the experimental data of Hall [2], we assume that the simulated concretes are made of coarse 
aggregate particles embedded in a mortar that has a sorptivity and saturated water content of 2.57 $\mathrm{mm} / \mathrm{min}^{0.5}$ and 0.27 respectively. Thus, the scaled sorptivity $\mathrm{s}$ of the mortar is $9.52 \mathrm{~mm} / \mathrm{min}^{0.5}$. We note however, that the choice of $s$ does not influence the simulated $S / S_{m}$ ratio as discussed in the preceding paragraph.

\subsection{Effect of aggregate volume fraction}

Fig. 6 shows the simulated water penetration profiles of concrete containing 10\%, 30\% and 50\% volume fraction of spherical coarse aggregate. The results are compared against the profile for mortar ( $0 \%$ coarse aggregate) which is essentially the same as that shown in Fig. $4 \mathrm{c}$ for $n=6$. It can be seen that the Boltzmann plots for concrete retains a characteristic steep-fronted profile. As expected, the absorbed water content decreases with increase in aggregate fraction since the aggregate particles are non-porous compared to the cement paste. Thus, the addition of aggregate particles dilutes the volume of the porous media through which absorption can occur. It is interesting to note that the difference in the absorbed water in concrete relative to mortar is initially proportional to the coarse aggregate fraction, but the difference gradually increases with penetration depth. The absorbed water profiles show a significant shift to the left as the aggregate concrete increases.

The shift in the penetration profile is due to redirection of the flow around aggregate particles and the resultant increase in tortuosity of the transport path. Thus, the required time for absorbed water to penetrate a certain depth increases with increase in aggregate volume fraction. This effect can be seen by comparing Fig. 7a and Fig. 7b, which show the distribution of water content in a threedimensional mesostructure of mortar and concrete respectively subjected to capillary absorption from the left face. Water does not penetrate into concrete as a sharp front parallel to the exposed surface, but advances in an uneven profile across the sample. The wetting front in concrete lags behind that of the mortar at every time step.

It should be noted that two-dimensional models are able to capture the dilution effect accurately, but not the tortuosity effect. This error increases with increase in the number of aggregate particles, aggregate fraction and aspect ratio. Obviously, a three-dimensional model represents the internal structure of concrete more realistically.

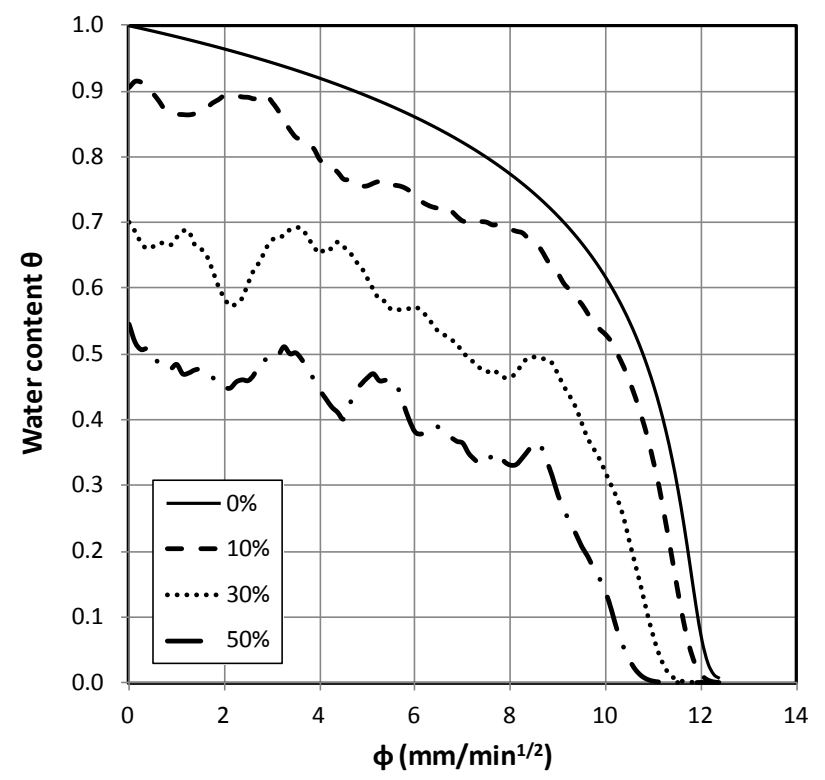

Fig. 6 Effect of aggregate volume fraction on water penetration profiles of concrete with 5-15 $\mathrm{mm}$ spherical coarse aggregate particles (Fuller-Thompson). 
a)
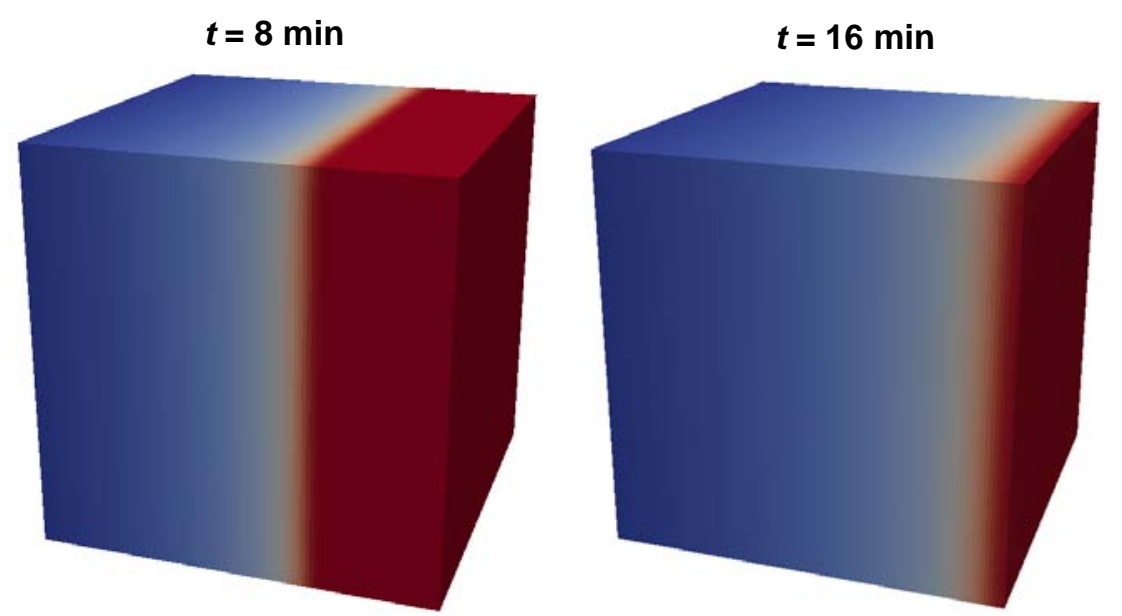

b)
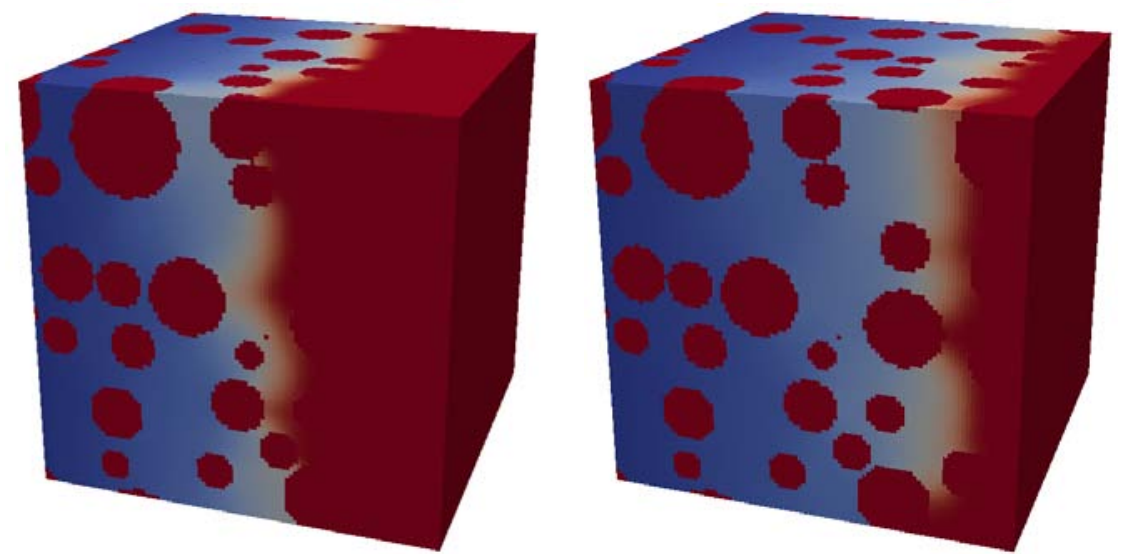

c)
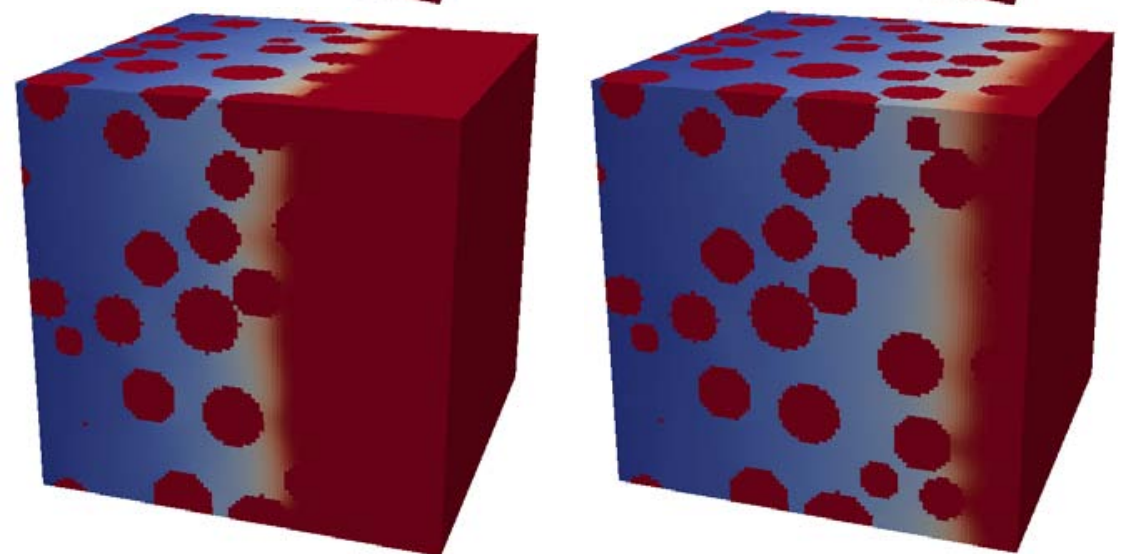

d)
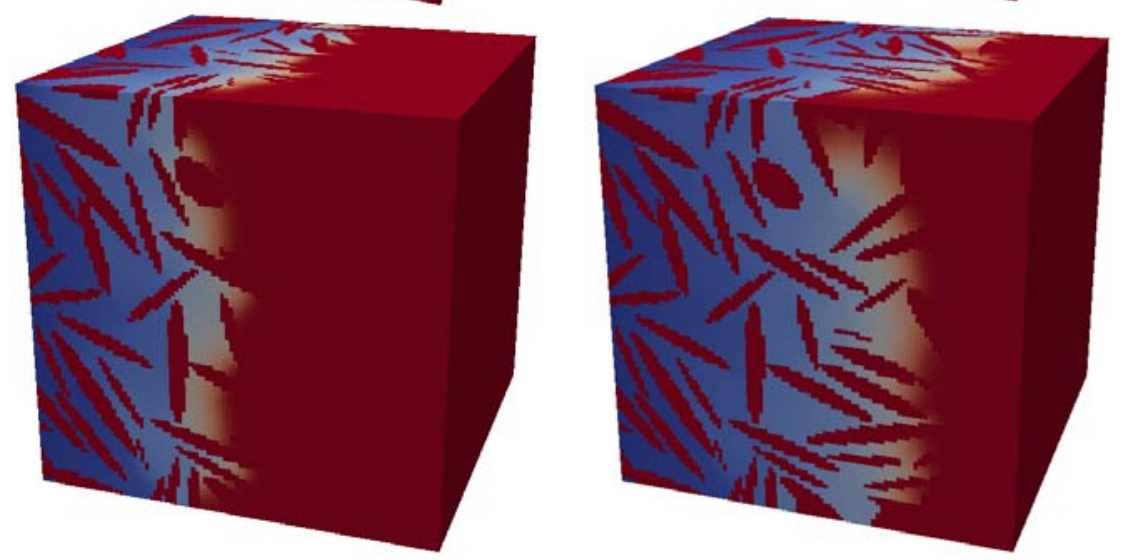

$\theta$

Fig. 7 Water penetration profiles at $t=8 \mathrm{~min}$ (left) and $t=16 \mathrm{~min}$ (right) for a $50^{3} \mathrm{~mm}^{3}$ sample at $0.5 \mathrm{~mm}$ voxel size containing no coarse aggregate (a) and $30 \%$ volume fraction of $5-15 \mathrm{~mm}$ spherical aggregate (b), 5-10 mm spherical aggregate (c) and 5-10 mm oblate spheroidal aggregate with aspect ratio $2: 2: 0.25$ (d). 
Another interesting observation from Fig. 6 is the fluctuation in the simulated penetration profiles for concrete in comparison to the mortar. It should be noted that the water contents at each depth are averaged over the whole section perpendicular to the direction of the flow. Thus, a source of the fluctuation is due to variation in the aggregate content at each depth. This has been observed in NMR measured water penetration profiles for concrete [24]. Part of the fluctuation can also be attributed to bending of the flow path around aggregate particles as can be seen in Fig. 7b. In our simulations, the amount of fluctuation increases with aggregate content, but this will probably smooth out if the computational cube size is increased.

The area under the water penetration profiles in Fig. 6 reflects the sorptivity of the sample. Fig. 8 shows the sorptivity of concrete normalised to the sorptivity of mortar and plotted against aggregate volume fraction. The results show that increasing aggregate fraction decreases the sorptivity of concrete. However, the normalised sorptivity is always less than $\left(1-V_{a}\right)$, where $V_{a}$ is the aggregate volume fraction, and this discrepancy increases with aggregate content due to the tortuosity effect. The simulated sorptivity values are also in good agreement with the analytical equation from Hall et al. [8] who showed that the effect of non-sorptive inclusions can be expressed using an extended version of the classical Maxwell effective medium approximation:

$$
\frac{S}{S_{m}}=1-1.25 V_{a}+0.26275 V_{a}^{2}
$$

This equation assumes that the inclusions are spherical, well-dispersed and uniformly distributed in a porous matrix. The inclusions can have any size distribution as long as the particles are large in comparison to the pores in the homogeneous matrix. It is also assumed that the presence of the inclusions does not modify the pore structure of the matrix. However, the expression is exact for dilute systems only, and this explains a small discrepancy with our simulated sorptivity at high aggregate fractions.

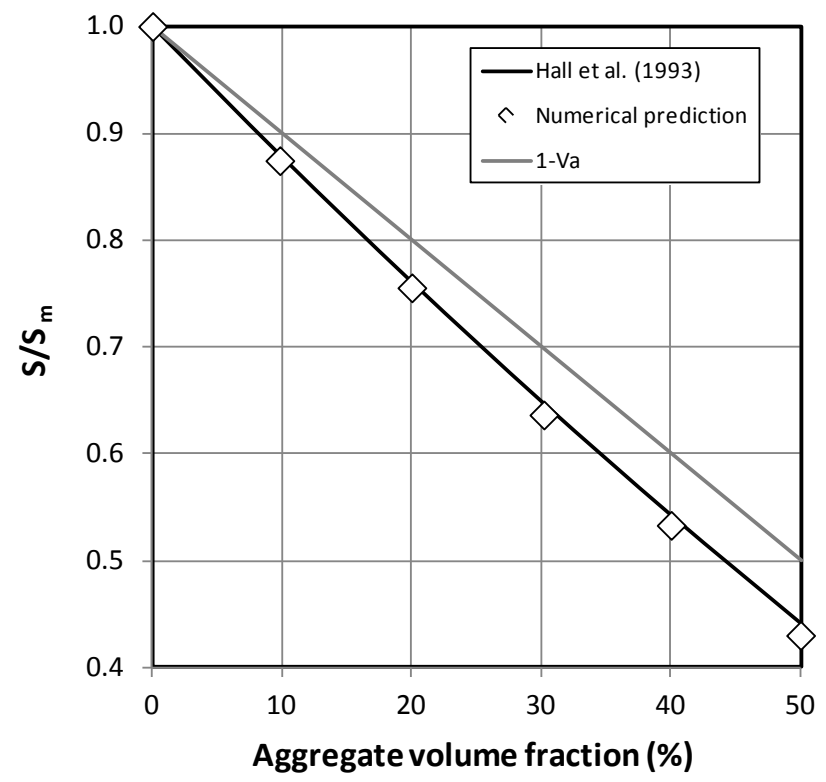

Fig. 8 Effect of coarse aggregate volume fraction on the normalised sorptivity $S / S_{m}$ of concrete containing 5-15 mm spherical aggregate particles.

\subsection{Effect of aggregate size}

Fig. 9 shows the effect of aggregate size distribution on the water penetration profile and the normalised sorptivity for concrete containing spherical aggregate particles. The results show that the fluctuation in the profiles increased slightly with aggregate size. Because the simulations were carried out whilst maintaining the computational cube constant at $50^{3} \mathrm{~mm}^{3}$, the variation in aggregate content at each depth is expected to increase with aggregate size. Interestingly, no significant horizontal shift was observed at the wetting front suggesting that changing particle size distribution makes little difference to the tortuosity of the flow path. 
It should be noted that the simulation shown in Fig. 9a is carried out at a constant aggregate fraction. Increasing aggregate size distribution necessitates a reduction in the number of aggregate particles to maintain constant volume fraction in the sample. This shows that the effect of the additional bending of flow around larger aggregate particles is counter balanced by the reduction in the number of aggregate particles. Fig. 9b shows that the size distribution of aggregate particles has a negligible influence on the sorptivity of concrete.

These features can also be seen by comparing Fig. 7b and Fig 7c, which shows the distribution of water content in a three-dimensional mesostructure containing 5-15 mm and 5-10 mm spherical particles respectively. The mesostructure containing larger aggregate size shows a highly irregular water content distribution, but no significant lag in the depth of the wetting front.

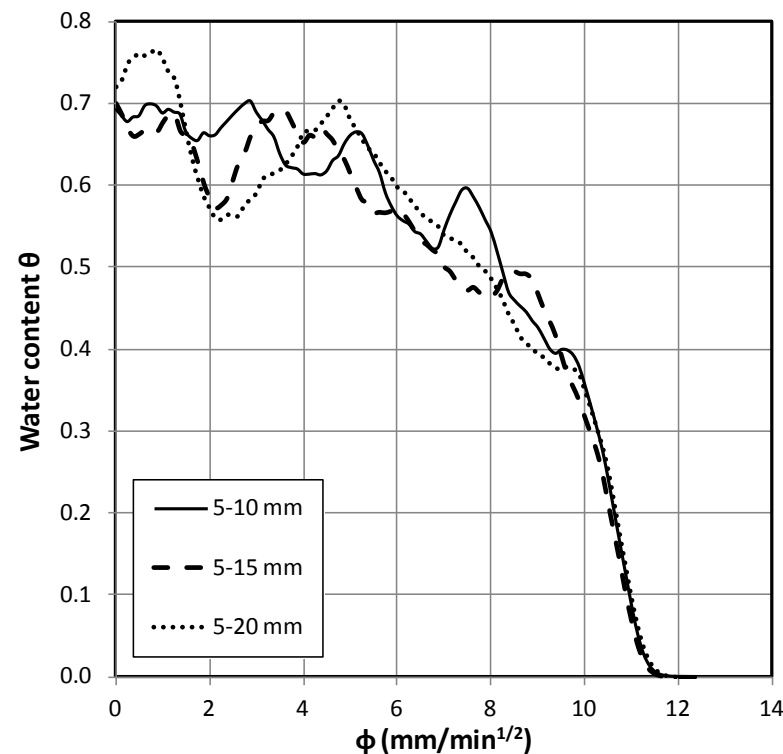

(a)

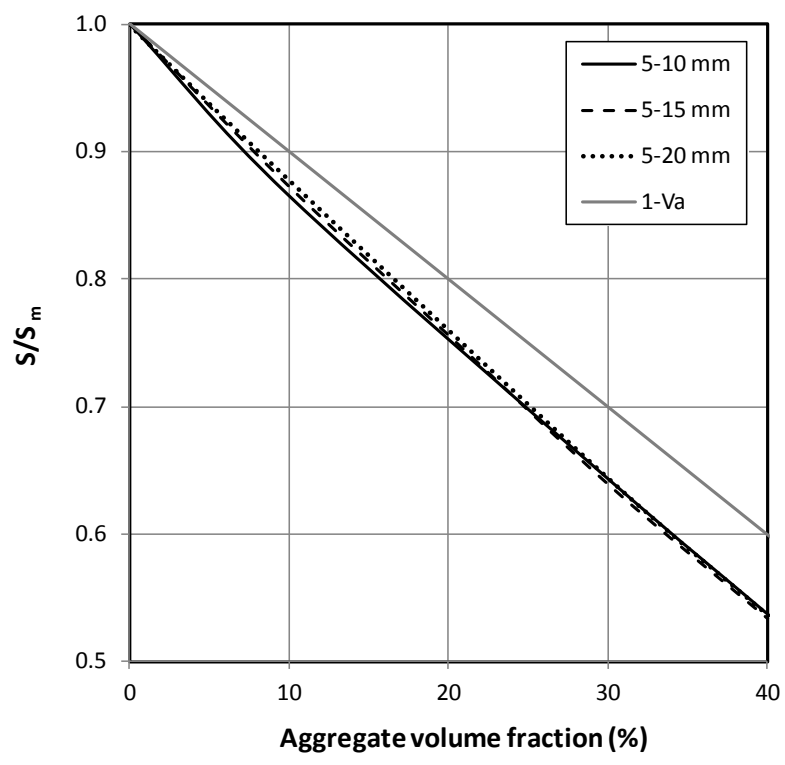

(b)

Fig. 9 Effect of aggregate size distribution on water penetration profiles in concrete containing $30 \%$ vol. spherical coarse aggregate particles (a) and normalised sorptivity $S / S_{m}$ of concrete containing a range of coarse aggregate fraction (b).

\subsection{Effect of aggregate shape}

Seven particle shapes were examined (Table 1). The aspect ratios of these were chosen to maintain a constant particle volume so that for a sample containing a certain volume fraction of aggregate particles, the total number of aggregate particles will not be influenced by a change in aggregate shape. The results plotted in Fig. 10 and Fig. 11 show that aggregate particle shape has a significant influence on the water penetration profiles and the sorptivity of concrete. Simulations carried out on samples containing ellipsoidal aggregates show a significant shift in the water penetration profile and a drop in sorptivity when compared to spherical aggregates. This can also be observed by comparing Fig. 7c and Fig. 7d, which shows that the sample containing ellipsoidal particles consistently achieves lower penetration depths compared to the sample containing spherical aggregate particles.

In all cases, the simulated sorptivity decreased when spherical aggregate are replaced by ellipsoidal ones (Fig. 11). The effect is most significant for oblate spheroidal, followed by tri-axial ellipsoidal and prolate spheroidal aggregate particles. Furthermore, the effect becomes more severe with the increase in aspect ratio. The decrease in sorptivity is also more significant at higher aggregate fractions. At $40 \%$ coarse aggregate fraction, the sorptivity for concrete with ellipsoidal aggregate is up to $16 \%$ lower than that for spherical aggregate. These findings are in good agreement with our previous study on the effect of aggregate shape on the diffusion of mortar and concrete [15]. 


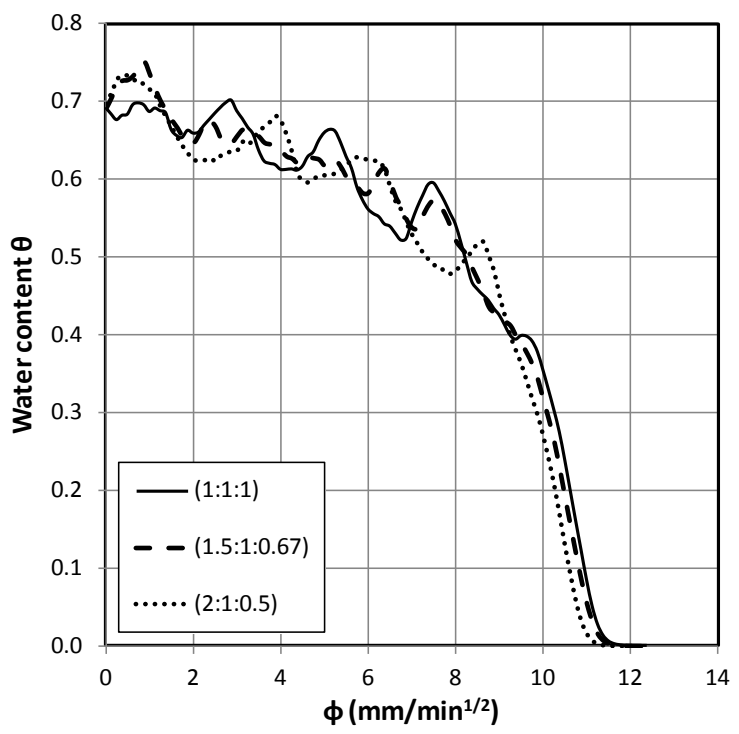

a) Tri-axial ellipsoidal $(a>b>c)$

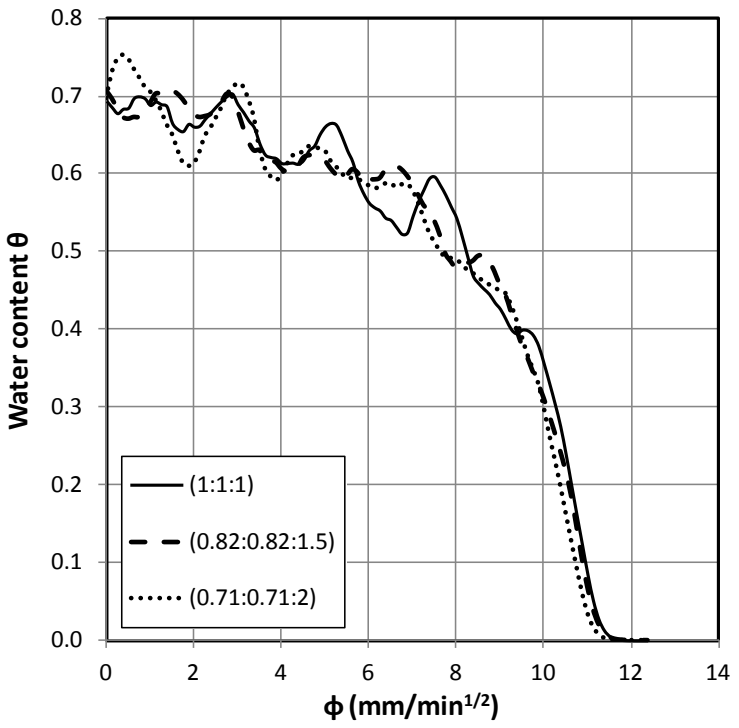

b) Prolate spheroidal $(\mathrm{a}=\mathrm{b}<\mathrm{c})$

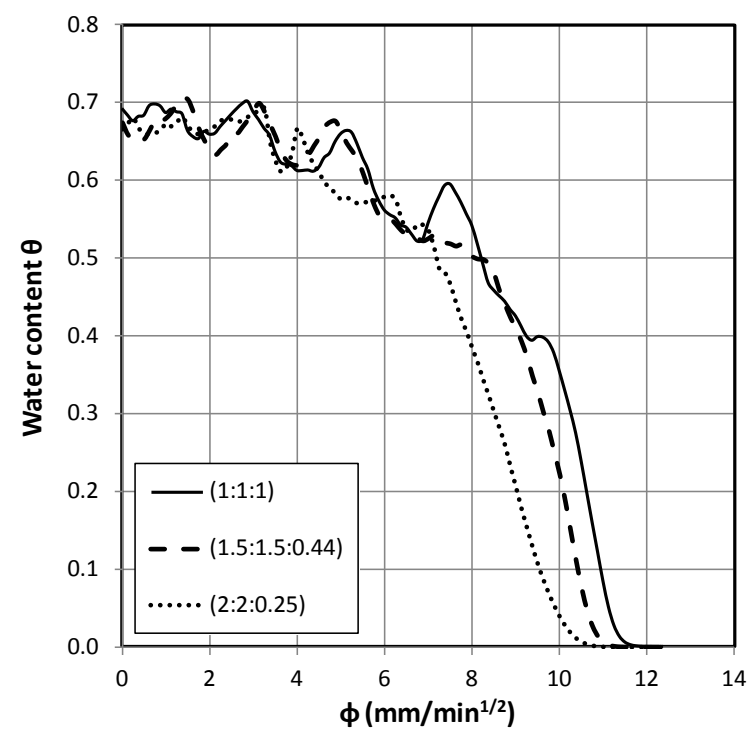

c) Oblate spheroidal $(a=b>c)$

Fig. 10 Effect of aggregate shape on water penetration profiles in concrete containing $30 \%$ vol. coarse aggregate particles (aggregate gradation $=5-10 \mathrm{~mm}$, Fuller-Thompson) 


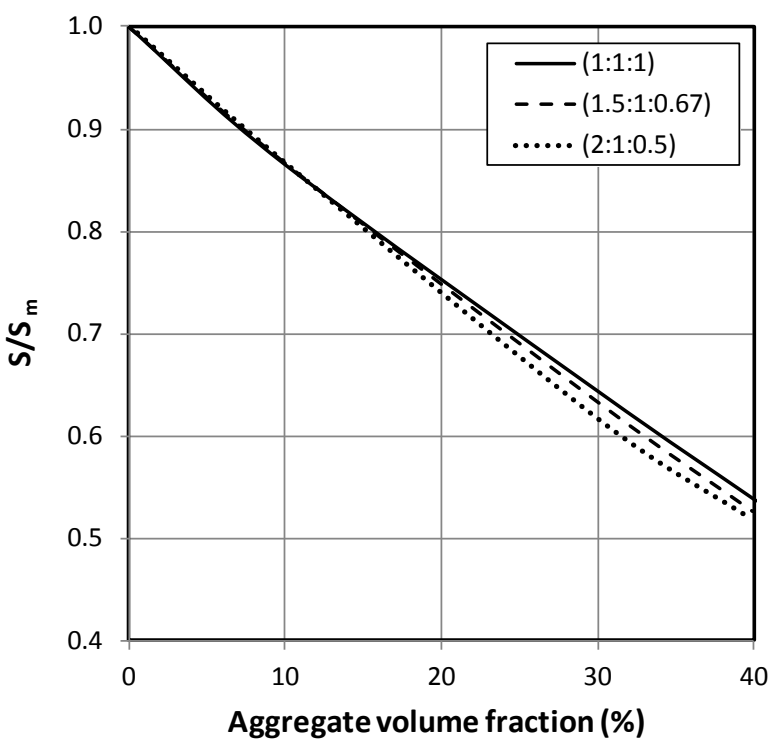

a) Tri-axial ellipsoidal $(a>b>c)$

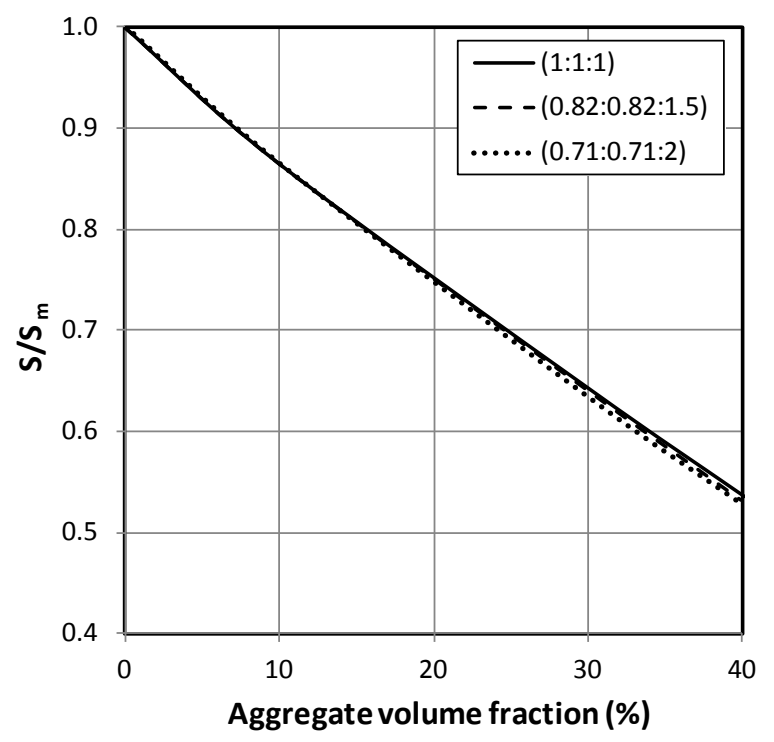

b) Prolate spheroidal $(\mathrm{a}=\mathrm{b}<\mathrm{c})$

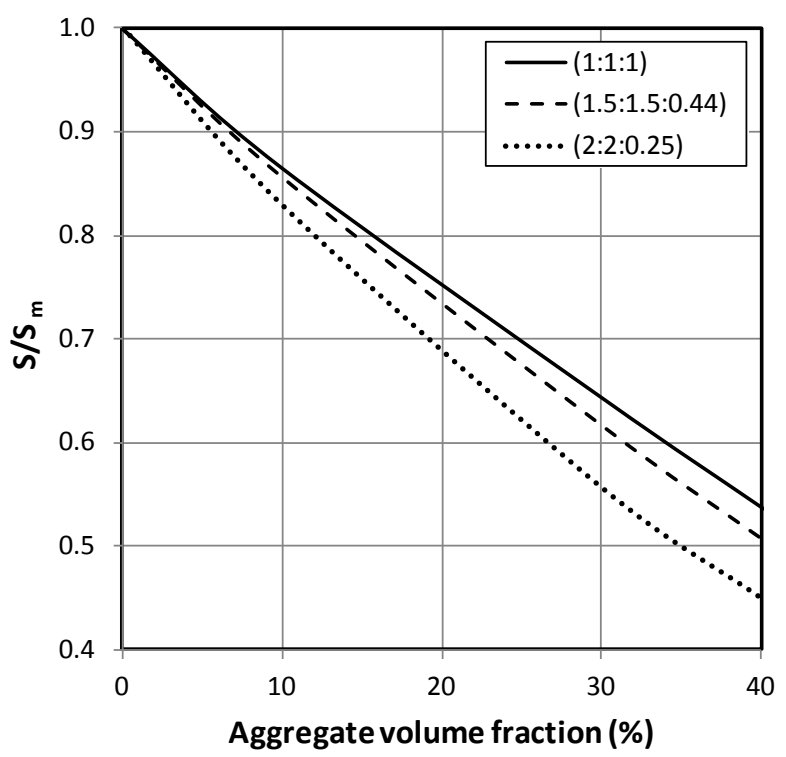

c) Oblate spheroidal $(\mathrm{a}=\mathrm{b}>\mathrm{c})$

Fig. 11 Effect of aggregate shape on the normalised sorptivity $S / S_{m}$ of concrete containing a range of aggregate volume fraction (aggregate gradation $=5-10 \mathrm{~mm}$, Fuller-Thompson)

\section{Discussion}

As mentioned earlier, the validity of Eq. 3 to predict water penetration profiles in bricks, stones and mortars has been tested in numerous studies based on comparison against measured wetting profiles, e.g. refs [1, 9, 24-27]. Therefore, it is encouraging to see that our simulation for mortar (Fig. 4) matches experimental results very well. The approximate exponential function for hydraulic diffusivity (Eq. 5) in which $D_{0}$ is obtained from sorptivity and $n$ is taken as 6 also seems to work well for mortars. The main advantage of this approach is that hydraulic diffusivity can be calculated from sorptivity, which is relatively easy to measure in the lab. However, there exists very little experimental data of water penetration profiles for concrete that we can compare our simulations against. Clearly more work is needed to confirm the validity of these equations and the assumptions therein for concrete. 
It is also interesting to note the agreement between the simulated sorptivity of concrete (Fig. 8) with that of Hall's equation [8]. This lends support to the use of Hall's equation as a quick means to estimate the sorptivity of concrete based on knowledge of the aggregate content and sorptivity of the matrix. It should be mentioned that although this equation was derived for homogeneous media containing a dilute amount of spherical inclusions, its validity seems to extend beyond the dilute regime provided properties are averaged over a representative volume, as it has been shown for gypsum plaster containing up to $49 \%$ vol. sand particles [8]. However, this is not the case for nonspherical inclusions. Indeed, our simulations (Fig. 11) show that the deviation from Hall's equation increases as the aggregate particles become increasingly non-spherical at aggregate fractions typical of concrete.

The model described here is useful for evaluating the effects of various parameters on capillary absorption that are difficult to isolate and quantify by laboratory measurements alone. However, the microstructure of real concrete is more complicated than what is captured at present by our model. The samples tested in this study are isotropic since aggregate particles were placed randomly in the computational cube. In real structures, various factors could lead to anisotropy such as segregation that occurs during transport, placement or compaction of concrete. These will further augment the influence of aggregate particles on the water absorption process.

It is also well-known that the presence of aggregate particles disturbs the microstructure of the cement paste, but this was not captured in our model. The paste region surrounding each aggregate particle, i.e. the interfacial transition zone (ITZ), contains on average higher porosity compared to paste region farther away and so it is reasonable to assume that water absorption will be accelerated, at least locally within the ITZ. However, the porosity and width of the ITZ is highly variable [31, 32] and the presence of porous ITZ is accompanied by a denser bulk cement paste because of water conservation in the microstructure. Thus, compared to other factors such as w/c ratio, degree of hydration and aggregate fraction, the net influence of ITZ on transport properties is not significant, as shown in several experimental $[2,5,33,34]$ and theoretical studies $[15,22,35,36]$. For example, the recent study by Wang \& Ueda [22] found only a small increase in absorbed water content even when the hydraulic diffusivity of the ITZ was assumed to be ten times greater than the bulk paste, and the size of the ITZ was tripled from 20 to $60 \mu \mathrm{m}$. Therefore, our approximation is not likely to have a huge impact on the estimated sorptivity.

Concretes also contain air voids that are either inadvertently entrapped because of incomplete compaction, or deliberately entrained. It is often assumed that these contribute little to transport since they appear as isolated voids, but they are in fact interconnected by capillary/gel pores. It has been shown that $10 \%$ air content can increase diffusivity and permeability by up to a factor of 2-3 [37]. However, air voids exert a much smaller capillary suction compared to capillary pores and rapidly become encircled/trapped by the advancing wetting front. Therefore, a reduction in sorptivity with increase in air content is usually observed for example in foamed/aerated concrete [38]. Here, the effect of air voids is to dilute the paste content and increase the tortuosity of the transport path, consistent with the effect of aggregate particles as modelled in this study.

Cracks, whether induced by mechanical loading, thermal gradient or drying shrinkage, are expected to influence the absorbed water profile $[4,6]$. The magnitude of the effect will depend on the width, geometry, spacing and connectivity of the cracks, the dissolution and precipitation of hydration products, and potential blockage/healing of the cracks with repeated wetting. This is a complex subject that is not well understood. There are also conflicting reports in the literature. For example, some studies suggest that load-induced microcracks can increase sorptivity significantly [39], while others found that the increase occurs only on a local level and not on the overall water absorption [40]. Other studies found that drying-induced microcracks have less influence on sorptivity compared to permeability $[2,41]$. Clearly, more work is needed to better understand these processes. An ongoing extension of the current model is to simulate cracks in the mesostructure and investigate the influence of cracks on transport. Treating the lattice elements as conductive pipes may be a simplified approach, but when combined with lattice-based fracture analysis [42], we expect the model to provide new insights into transport properties of concrete.

To improve the model's predictive capability, capillary absorption needs to be simulated at the pore scale to generate data that could then be used as input at the mesoscale. However, there is a lack of work in this area compared to, for example, pore-scale modelling of permeability [43-45]. More work is needed to characterise the connectivity of the pore structure and crack network in three-dimensions [46-48] to advance this area. However, none of the existing three-dimensional imaging techniques are able to image a representative volume of concrete at sufficiently high resolution to capture the pores 
and cracks important to transport. Furthermore, in order to model any deterioration of concrete, one needs to couple transport phenomena to chemical reactions and mechanical damage. The microstructural changes that occur for example due to dissolution and precipitation of products, need to be considered.

\section{Conclusions}

Capillary absorption in concrete was investigated numerically using a three-dimensional mesoscale model. Concrete was idealised as a heterogeneous composite of aggregate particles randomly placed in a porous matrix and discretised into a network of lattice elements. A non-linear finite element method was used to solve the governing diffusion equation that describes capillary absorption according to unsaturated flow theory. The distribution of absorbed water content in the mesostructure was calculated as a function of space and time, based on hydraulic diffusivity determined from the initial and saturated moisture content, and sorptivity of the porous matrix. The model was validated by comparing the simulated water penetration profile in mortar with available experimental data and analytical approximations. The model was then used to investigate the effect of heterogeneities produced by coarse aggregate particles on capillary absorption in concrete. The results showed that water does not penetrate into concrete as a sharp front parallel to the exposed surface, but advances in an uneven manner that is influenced by the amount, spatial distribution and shape of the aggregate particles. The wetting front in concrete lagged behind that of the mortar at each time step due to bending of flow around coarse aggregate particles and the increased tortuosity of the flow path. Thus, the required time for absorbed water to penetrate a certain depth increased. The shape of aggregate particles may have a significant effect on the water penetration profile and sorptivity. Replacing spherical aggregates with ellipsoidal aggregates produced a shift in the wetting front and a decrease in sorptivity. This is most significant for oblate spheroidal, followed by tri-axial ellipsoidal and prolate spheroidal aggregate particles. Furthermore, the effect increased with aspect ratio and aggregate volume fraction. However, aggregate size distribution had little influence on the water penetration profile and sorptivity because the increased tortuosity due flow around larger aggregate particles is balanced by the reduction in the number of aggregate particles. The modelling approach described in this paper is particularly useful for isolating and studying the influence of various parameters on capillary absorption that would otherwise be difficult to achieve through laboratory-based testing.

\section{Acknowledgments}

The research leading to these results has received funding from the European Union Seventh Framework Programme (FP7 / 2007-2013) under grant agreement 264448.

\section{References}

[1] C. Hall, Magazine of Concrete Research, 41 (1989) 51-61.

[2] H.S. Wong, M. Zobel, N.R. Buenfeld, R.W. Zimmerman, Magazine of Concrete Research, 61 (2009) 571-589.

[3] N.S. Martys, C.F. Ferraris, Cement and Concrete Research, 27 (1997) 747-760.

[4] D. Gardner, A. Jefferson, A. Hoffman, Cement and Concrete Research, 42 (2012) 972-981.

[5] N.R. Buenfeld, E. Okundi, Magazine of Concrete Research, 50 (1998) 339-351.

[6] J. Carmeliet, J.F. Delerue, K. Vandersteen, S. Roels, Int. J. Numer. Anal. Methods Geomech., 28

(2004) 671-687.

[7] W.J. McCarter, H. Ezirim, M. Emerson, Magazine of Concrete Research, 44 (1992) 31-37.

[8] C. Hall, W.D. Hoff, M.A. Wilson, J. Phys. D-Appl. Phys., 26 (1993) 31-34.

[9] D. Lockington, J.Y. Parlange, P. Dux, Materials and Structures, 32 (1999) 342-347.

[10] M. Wang, N. Pan, Mater. Sci. Eng. R-Rep., 63 (2008) 1-30.

[11] S. Shahbeyk, M. Hosseini, M. Yaghoobi, Computational Materials Science, 50 (2011) 1973-1990.

[12] S. Ai, L. Tang, Y. Mao, Y. Pei, Y. Liu, D. Fang, Computational Materials Science, 67 (2013) 133-139.

[13] E.J. Garboczi, D.P. Bentz, Advanced Cement Based Materials, 8 (1998) 77-88.

[14] L. Wang, T. Ueda, Computers and Concrete, 8 (2011) 125-142. 
[15] S. Dehghanpoor Abyaneh, H.S. Wong, N.R. Buenfeld, Computational Materials Science, 78 (2013) 63-73.

[16] Y.P. Xi, Z.P. Bazant, J. Mater. Civ. Eng., 11 (1999) 58-65.

[17] A. Ababneh, F. Benboudjema, Y.P. Xi, J. Mater. Civ. Eng., 15 (2003) 183-191.

[18] E. Samson, J. Marchand, Computers \& Structures, 85 (2007) 1740-1756.

[19] S. Kamali-Bernard, F. Bernard, W. Prince, Computational Materials Science, 45 (2009) 528-535.

[20] F. Bernard, S. Kamali-Bernard, Computational Materials Science, 61 (2012) 106-115.

[21] H. Sadouki, J.G.M. van Mier, Materials and Structures, 30 (1997) 579-587.

[22] L. Wang, T. Ueda, Ocean Engineering, 38 (2011) 519-528.

[23] C. Hall, Materials and Structures, 27 (1994) 291-306.

[24] C. Leech, D. Lockington, P. Dux, Materials and Structures, 36 (2003) 413-418.

[25] R.J. Gummerson, C. Hall, W.D. Hoff, R. Hawkes, G.N. Holland, W.S. Moore, Nature, 281 (1979) 5657.

[26] J.-F. Daian, Transport in Porous Media, 3 (1988) 563-589.

[27] T.A. Carpenter, E.S. Davies, C. Hall, L.D. Hall, W.D. Hoff, M.A. Wilson, Materials and Structures, 26 (1993) 286-292.

[28] W.B. Fuller, S.E. Thompson, Trans. Am Soc Civ Eng, 59 (1907) 67-143.

[29] X. Jia, R.A. Williams, Powder Technology, 120 (2001) 175-186.

[30] J.E. Bolander Jr, S. Berton, Cement and Concrete Composites, 26 (2004) 861-871.

[31] K.L. Scrivener, A.K. Crumbie, P. Laugesen, Interface Science, 12 (2004) 411-421.

[32] H.S. Wong, N.R. Buenfeld, Cement and Concrete Research, 36 (2006) 1091-1097.

[33] A. Delagrave, J. Marchand, M. Pigeon, Advanced Cement Based Materials, 7 (1998) 60-65.

[34] J.D. Shane, T.O. Mason, H.M. Jennings, E.J. Garboczi, D.P. Bentz, Journal of the American Ceramic Society, 83 (2000) 1137-1144.

[35] D.P. Bentz, E.J. Garboczi, E.S. Lagergren, Cement Concrete and Aggregates, 20 (1998) 129-139.

[36] J.-J. Zheng, H.S. Wong, N.R. Buenfeld, Cement and Concrete Research, 39 (2009) 805-813.

[37] H.S. Wong, A.M. Pappas, R.W. Zimmerman, N.R. Buenfeld, Cement and Concrete Research, 41 (2011) 1067-1077.

[38] E.K.K. Nambiar, K. Ramamurthy, Cement and Concrete Research, 37 (2007) 1341-1347.

[39] M. Şahmaran, V. Li, Materials and Structures, 42 (2009) 593-603.

[40] Z.F. Yang, W.J. Weiss, J. Olek, J. Mater. Civ. Eng., 18 (2006) 424-434.

[41] H.S. Wong, N.R. Buenfeld, J. Hill, A.W. Harris, Advances in Cement Research, 19 (2007) 35-46.

[42] P. Grassl, H.S. Wong, N.R. Buenfeld, Cement and Concrete Research, 40 (2010) 85-93.

[43] M. Zalzale, P.J. McDonald, Cement and Concrete Research, 42 (2012) 1601-1610.

[44] M. Zhang, G. Ye, K.v. Breugel, Computational Materials Science, 68 (2013) 142-151.

[45] H.S. Wong, R.W. Zimmerman, N.R. Buenfeld, Cement and Concrete Research, 42 (2012) 476-483.

[46] L. Holzer, P. Gasser, B. Muench, Quantification of capillary pores and Hadley grains in cement paste using FIB-nanotomography, 2006.

[47] M.K. Head, H.S. Wong, N.R. Buenfeld, Cement and Concrete Research, 36 (2006) 1483-1489.

[48] E. Gallucci, K. Scrivener, A. Groso, M. Stampanoni, G. Margaritondo, Cement and Concrete

Research, 37 (2007) 360-368. 